\title{
Association Between Decreased Srpk3 Expression And An Increase In Substantia Nigra Alpha- Synuclein Levels In An MPTP-Induced Parkinson's Disease Mouse Model
}

\section{Min Hyung Seo}

Sangji University https://orcid.org/0000-0003-4709-203X

Sujung Yeo ( $\sim$ pinkteeth@naver.com )

Sangji University https://orcid.org/0000-0001-9883-6153

\section{Research Article}

Keywords: Parkinson's disease, Srpk3, a-synuclein, dopaminergic neuron, tyrosine hydroxylase

Posted Date: January 11th, 2022

DOI: https://doi.org/10.21203/rs.3.rs-1207279/v1

License: (c) (1) This work is licensed under a Creative Commons Attribution 4.0 International License. Read Full License 


\section{Abstract}

Parkinson's disease (PD) is known as the second most common neurodegenerative disease, which is caused by destruction of dopaminergic neurons in the substantia nigra (SN) of the brain; however, the reason for the death of dopaminergic neurons remains unclear. An increase in a-synuclein (a-syn) is considered an important factor in the pathogenesis of PD. In the current study, we investigated the association between PD and serine/arginine-rich protein specific kinase 3 (Srpk3) in MPTP-induced parkinsonism mice model and in SH-SY5Y cells treated with MPP+. Srpk3 expression was significantly downregulated, while tyrosine hydroxylase $(\mathrm{TH})$ decreased and a-synuclein (a-syn) increased after 4 weeks of MPTP intoxication treatment. Dopaminergic cell reduction and a-syn increase were demonstrated by inhibiting Srpk3 expression by siRNA in SH-SY5Y cells. Moreover, a decrease in Srpk3 expression upon siRNA treatment promoted dopaminergic cell reduction and a-syn increase in SH-SY5Y cells treated with MPP+. These results suggest that the decrease in Srpk3 expression due to Srpk3 siRNA caused both a decrease in TH and an increase in a-syn. This raises new possibilities for studying how Srpk3 controls dopaminergic cells and a-syn expression, which may be related to the pathogenesis of PD. Our results provide an avenue for understanding the role of Srpk3 during dopaminergic cell loss and a-syn increase in the SN. Furthermore, this study could support a therapeutic possibility for PD in that the maintenance of Srpk3 expression inhibited dopaminergic cell reduction.

\section{Introduction}

Parkinson's disease (PD) is the second most common neurodegenerative disorder after Alzheimer's disease. PD is characterized by behavioral symptoms such as tremors, bradykinesia, akinesia, and stiffness. PD is caused by the loss of dopaminergic neurons in the substantia nigra (SN) [1, 2]; however, the reason for the death of dopaminergic neurons remains unclear. Neurodegeneration in PD has been reported to be correlated with the occurrence of Lewy bodies, which are intracellular inclusions containing aggregates of the disordered protein a-synuclein (a-syn) [3]. An increase in a-syn is considered an important factor in the pathogenesis of PD.

In this study, we analyzed altered gene expression levels in SN regions in a mouse model of PD intoxicated with 1-methyl-4-phenyl-1,2,3,6-tetrahydropyridine (MPTP) to induce dopaminergic cell reduction [4]. Through the application of an automated polymerase chain reaction (PCR)-based expression profiling system with high sensitivity and reliability [5], several genes whose expression was altered in the neurodegenerative conditions caused by MPTP injection were detected. In addition, serine/arginine-rich protein specific kinase 3 (Srpk3) was significantly downregulated after 4 weeks of MPTP treatment.

Srpk3 belongs to the serine/arginine protein kinase family and is controlled by a muscle-specific enhancer regulated by MEF2 [6]. Srpk3 is expressed in the lung, skin, spleen, heart, joint, muscle, and brain. This gene encodes a protein kinase that is specific for the serine/arginine-rich domain (SR domain) 
family of splicing factors [6], which has been reported to phosphorylate serine/arginine repeat-containing proteins [6].

These intriguing observations lead to the hypothesis that a decrease in Srpk3 might be related to PD. In the pathogenesis of PD, dopaminergic cell reduction, which is related to a-syn increase in mice intoxicated with MPTP, could be affected by the decrease in Srpk3 levels. To verify this hypothesis, we observed changes in Srpk3 expression associated with decreased tyrosine hydroxylase (TH) expression and increased a-syn in a chronic MPTP-induced PD mouse model. Moreover, by suppressing the expression of Srpk3 by siRNA in SH-SY5Y cells, the decrease in dopaminergic cell and the increase in asyn were investigated.

In this regard, the aim of our research is to investigate the association between Srpk3 and PD, and to provide an insight into the role of Srpk3 during dopaminergic cell loss and a-syn increase in the SN. Furthermore, this study could support the therapeutic potential of PD in that maintenance of Srpk3 expression inhibits dopaminergic cell decline.

\section{Materials And Methods}

\section{MPTP model of Parkinson's disease}

Six-week-old male C57BL/6 mice (20-22 g; DBL, Korea) were divided into two groups: the control group (CTL) and the MPTP intoxicated group (MPTP). Mice in the CTL were injected with $0.9 \%(100 \mu \mathrm{L})$ saline intraperitoneally once daily for 4 weeks, and mice in the MPTP were injected with MPTP-HCl $(20 \mathrm{mg} / \mathrm{kg}$ of free base) in $0.9 \%(100 \mu \mathrm{L})$ saline intraperitoneally once daily for 4 weeks to produce a sustained chronic parkinsonism mouse model. On the day after the final MPTP treatment, the mice were anesthetized using Alfaxan and perfused with $0.05 \mathrm{M}$ sodium phosphate buffer (PBS). The Sangji University Animal Experimentation Committee approved all animal protocols used in this study. Guidelines around animal experiments have been followed. All reagents used in the study were purchased from Sigma (USA) unless otherwise stated.

\section{Rna Extraction And Microarray Analysis}

Total RNA was extracted from the bilateral SN using an RNeasy Plus Mini Kit (QIAGEN, USA) in. The quality of the isolated RNA was quantified using a NanoDrop ND-1000 spectrophotometer (NanoDrop Technologies, USA). An aliquot of the total RNA was subjected to an Affymetrix GeneChip ${ }^{\circledR}$ Mouse Gene 1.0 ST Array (Affymetrix, USA) according to the GeneChip Whole Transcript Sense Target Labeling Assay manual $[7,8]$.

\section{Microarray Data Analysis}


Quality control of the scanned data was confirmed using the Expression Console software (Affymetrix). Microarray data were analyzed using the GenPlex ver. 3.0 (ISTECH, Korea). The mean signal intensities of the genes were obtained from the two chips for each group. Following normalization, the genes that satisfied the conditions of Student's $t$-test significance criterion $(p<0.05)$ and the fold change cutoff [2] were identified using the differentially expressed genes (DEG)-finding module.

\section{Cell Lines And Cultures}

The neuroblastoma cell line SH-SY $5 Y$ was maintained under standard cell culture conditions $\left(37^{\circ} \mathrm{C}, 5 \%\right.$ $\mathrm{CO}_{2}$ ) in minimum essential medium (MEM; Welgen, Namcheon-myeon) containing $10 \%$ fetal bovine serum (FBS; BioWhittaker), $0.1 \mathrm{mM}$ nonessential amino acids, $100 \mathrm{U} / \mathrm{mL}$ of penicillin, and $100 \mathrm{mg} / \mathrm{mL}$ streptomycin.

\section{Short Interfering Rna (Sirna) Knockdown}

Stealth siRNA against Srpk3 (5- GAA AAC UGC CUG UUU GUU U -3) and negative control duplexes (i.e., scrambled siRNA against Srpk3, 5- UUC UCC GAA CGU GUC ACG UTT -3) were purchased from Bioneer Inc (Korea).

\section{Sirna Transfection}

The SH-SY5Y cells were incubated in Opti-MEM medium (Gibco, USA) for at least one day before performing siRNA transfection. The transfection reagent (Promega, USA) and Srpk3 siRNA were used (3.5:1) for transfection when the density of SH-SY5Y cells was $60 \%$. Transfection was continued for $24 \mathrm{~h}$.

\section{Mpp+ Treatment}

The SH-SY5Y cells were treated with $500 \mu \mathrm{M}$ MPP+ iodide (Sigma) for $18 \mathrm{~h}$.

\section{Immunohistochemistry}

After four weeks, the brains of mice were resected, fixed in PBS containing 4\% paraformaldehyde for $12 \mathrm{~h}$ at $4^{\circ} \mathrm{C}$, rinsed with PBS, immersed in $30 \%$ sucrose solution for $12 \mathrm{~h}$ at $4^{\circ} \mathrm{C}$, and then cryo-sectioned. Sections of the brains were cut using a cryomicrotome (30- $\mu \mathrm{m}$ thickness). Immunohistochemical analyses were performed using an $\mathrm{ABC}$ kit and a Mouse on Mouse (M.O.M) immunodetection kit (Vector Laboratories, CA) by a modification of the avidin-biotin-peroxidase method. Briefly, sections encompassing the entire striatum (ST) and SN were incubated in PBS with $3 \% \mathrm{H}_{2} \mathrm{O}_{2}$, exposed to $3 \%$ bovine serum albumin and $0.3 \%$ Triton $\mathrm{X}-100$ for $1 \mathrm{~h}$, and treated with an avidin biotin blocking kit (Vector 
Laboratories). Brain sections were treated with an M.O.M. mouse Ig-blocking reagent (Vector Laboratories) for $1 \mathrm{~h}$ at room temperature prior to incubation with the primary antibody. Each section was stained overnight with anti-TH (1:2000; Santa Cruz Biotechnology, USA), anti-Srpk3 (1:1000; Cloud-clone Corp., USA), and anti-a-syn (1:500; Santa Cruz Biotechnology, USA) antibodies at $4^{\circ} \mathrm{C}$. The sections were incubated with a biotinylated anti-mouse IgG, followed by an avidin-biotin-peroxidase complex, and then developed using a diaminobenzidine-hydrogen peroxide solution $(0.03 \%$ hydrogen peroxide and $0.003 \%$ 3,3-diaminobenzidine in $0.05 \mathrm{M}$ Tris, pH 7.0).

\section{Immunofluorescence}

The cells were treated using the same procedures as those used for immunohistochemistry. The cells were incubated with primary antibodies and then with biotinylated anti-mouse IgG and anti-rabbit IgG. The brain sections were treated with a fluorescein avidin DCS (Vector Laboratories, USA) for mouse antiTH (1:2,000) and rabbit anti-Srpk3 (1:1000). Thereafter, cells were treated with an avidin biotin blocking kit and an M.O.M mouse Ig blocking reagent (Vector Laboratories), followed by staining with anti-TH and anti-Srpk3 IgG at $4^{\circ} \mathrm{C}$ overnight. Each group was treated with a biotinylated anti-mouse IgG and antirabbit IgG, followed by incubation with fluorescein avidin D and rhodamine avidin D (Vector Laboratories). Photographic documentation was performed using a Nikon X-Cite-Series 120 Q microscope (Nikon, Japan). Images were collected using an ECLIPSE Ni-U microscope (Nikon) equipped with a digital camera (DS-Fi2; Nikon). Images were acquired using the imaging software NIS-Elements F ver. 4.00.00 (Nikon). Tiff images and quantification data were collected from each image data file using Image J software (NIH, USA).

\section{Western Blotting}

Western blotting

The ST and SN regions were homogenized using radioimmunoprecipitation assay buffer on ice for 30 min. After centrifugation at 12,000 rpm for $20 \mathrm{~min}$, the soluble supernatant samples were then collected, and equal concentrations of protein samples were separated using $12 \%$ sodium dodecyl sulfatepolyacrylamide gel electrophoresis and transferred to polyvinylidene difluoride membranes (Bio-Rad, USA). The membranes were blocked with $5 \%$ skim milk in $0.1 \%$ Tris-buffered saline (TBS) for $1 \mathrm{~h}$ at room

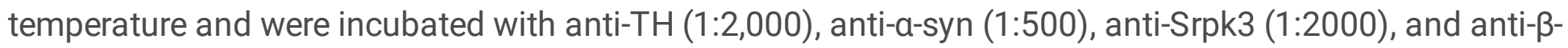
actin (1:5,000, Santa Cruz Biotechnology) antibodies. Thereafter, the membranes were washed with $0.1 \%$ TBS containing $0.1 \%$ Tween-20 (TBST) and incubated with an anti-mouse IgG-peroxidase antibody (1:2,000, Bio-Rad), and the antigen-antibody complexes were visualized using the Pierce ECL western blotting Substrate (Thermo Scientific, USA). The percentage of densitometric values for immunoreactive bands was calculated in each experiment. The density was measured using the ImageJ software (NIH).

\section{Statistical analysis}


Statistical analyses were performed using analysis of variance (ANOVA) and Student's $t$-test in SPSS 25 (SPSS Inc., USA). All values are presented as mean \pm standard error.

\section{Results}

To confirm that the MPTP-induced parkinsonism mouse model was well established, we evaluated changes in TH and a-syn expression. The TH levels were significantly lower in the MPTP group (Figure 1b and d) than in the CTL group (Figure 1a and c). Western immunoblot analysis also showed a significant decrease $(p<0.005)$ in the TH levels of the ST and SN of the MPTP group (Figure 1e). The level of a-syn in the SN region was significantly higher in the MPTP group (Figure 1f) than in the CTL group (Figure 1c). Western immunoblot analysis consistently showed a significant increase $(p<0.005)$ of a-syn levels in the MPTP group (Figure $1 \mathrm{~g}$ and $1 \mathrm{~h}$ ).

Srpk3 expression levels in the SN regions were noticeably lower in mice in the MPTP group than in mice in the CTL group (Figure 2). As seen in the immunoblot assay observations, the results of microarray analysis and western immune blot assay indicated that Srpk3 expression significantly decreased in MPTP, compared to the control (Figure $2 \mathrm{~h}$ ).

To investigate the relationship between Srpk3 and a-syn, we conducted experiments in which Srpk3 colocalized with a-syn in the SN regions of an MPTP-induced 4-week parkinsonism mouse model. In the control, Srpk3 expression was found to be co-localized with a-syn. In the MPTP group, co-localized Srpk3 and a-syn were decreased compared to the control. It is thought that even though a-syn increased in the MPTP group, decreased Srpk3caused decreased co-localization. This suggests that Srpk3 was not sufficient to cover a-syn in the MPTP group (Figure 3).

To investigate the relationship between decreased Srpk3 and increased a-syn, we conducted experiments in which Srpk3 expression was knocked down by using Srpk3 siRNA in SH-SY5Y cells. Srpk3 siRNA led to a decrease in Srpk3 expression but increase in a-syn expression in SH-SY5Y cells (Figure 4).

To investigate the effect of decreased Srpk3 on the PD state, we performed experiments in which Srpk3 expression was knocked down using Srpk3 siRNA in MPP + treated SH-SY5Y cells. Western blot analysis showed that decreased Srpk3 exacerbated tyrosine hydroxylase $(\mathrm{TH})$ reduction and a-syn increase in $\mathrm{MPP}+$ treated SH-SY5Y cells (Figure 5). Immunofluorescence analysis of Srpk3 and a-syn revealed colocalization, and Srpk3 expression was stronger in the CTL group (Figure 6b) than in the MPTP group (Figure 6f). Conversely, a-syn was more strongly expressed in the MPTP group than in the CTL group (Figure $6 \mathrm{c}$ and $\mathrm{g}$ ). Even in the merged images (Figure $6 \mathrm{~d}$ and $\mathrm{h}$ ), the expression of Srpk3 was stronger in the CTL group (Figure 6d), whereas that of a-syn was stronger in the MPTP group (Figure 6h).

\section{Discussion}

The TH levels were significantly lower in the MPTP group than in the CTL group. Moreover, the levels of asyn in the SN region was noticeably higher in the mice in the MPTP group than in the mice in the CTL 
group. These results verified the correct formation of the MPTP-induced parkinsonism mouse model.

In this study, the expression level of Srpk3 in the SN region was significantly lower in the mice in the MPTP group than in the mice in the CTL group. As shown in the immunohistochemistry observations, the results of microarray analysis and western immunoblot assay showed that Srpk3 expression was significantly reduced in the MPTP group.

To investigate the relationship between Srpk3 and a-syn, we conducted experiments in which Srpk3 colocalized with a-syn in the SN of an MPTP induced 4-week parkinsonism mouse model. In the control, Srpk3 expression was found to be co-localized with a-syn. In the MPTP group, co-localized Srpk3 and asyn were decreased compared to the control. It is thought that even though a-syn increased in the MPTP group, decreased Srpk3 caused decreased co-localization. In the control group, Srpk3, which was not colocalized with a-syn, was observed but not in the MPTP group. This suggests that Srpk3 was not sufficient to cover a-syn in the MPTP group.

We conducted experiments in which Srpk3 expression was knocked down using Srpk3 siRNA in SH-SY5Y cells. Srpk3 siRNA led to a decrease in Srpk3 expression, but increased a-syn expression in SH-SY5Y cells. This suggests that a decrease in Srpk3 expression is associated with an increase in a-syn expression.

To investigate the effect of decreased Srpk3 on the PD state, we carried out experiments in which Srpk3 expression was knocked down by using Srpk3 siRNA in MPP + treated SH-SY5Y cells, which was similar to the status of PD. Western immunoblot assay showed that the decrease in Srpk3 exacerbated the increase in a-syn in MPP + treated SH-SY5Y cells. These results suggest that the decrease in Srpk3 expression due to Srpk3 siRNA caused both the dopaminergic cell reduction and the a-syn increase. This raises a new avenue for studying how Srpk3 controls dopaminergic cells and a-syn expression, which may be related to the pathogenesis of PD.

Srpk3 phosphorylates its substrates at serine residues that are located in regions rich in arginine/serine dipeptides [6]. Several kinds of a-syn that are phosphorylated at serine have been reported to be involved in the pathogenesis of PD [9]. Based on these previous studies and our results, it is thought that Srpk3 might be involved in a-syn phosphorylation-related neurodegeneration of dopaminergic neurons. In previous research, striated muscles have been reported to be highly sensitive to the level of Srpk3 expression [10]. Srpk3-null mice displayed a new type 2 fiber-specific myopathy with a significant increase in centrally placed nuclei [10]. Myopathy in Srpk3 mutant mice is characterized by no signs of muscle degeneration or regeneration, such as myofiber disarray, muscle cell death, or neutrophil infiltration [10]. Combining these studies with our results suggests that motor dysfunction in PD might be associated with decreased Srpk3. In a previous study, we reported the decreased expression of Srpk3 in the muscles of MPTP-induced parkinsonian mice, which might be related to the increased expression of a-syn [11]. In this study, we confirmed a decrease in Srpk3 expression at both gene and protein levels in the substantia nigra-a major lesion site in PD. The decreased expression of Srpk3 in the brain might be one of the causes of dopaminergic cell degeneration since siRNA-induced decrease in Srpk3 expression led to an increase in a-syn expression in SH-SY5Y cells. 
In conclusion, our results demonstrated that Srpk3 expression was decreased in the MPTP parkinsonism mouse model and decreased Srpk3 expression increased a-syn levels in SH-SY5Y cells induced by Srpk3 siRNA. These results suggest an association between Srpk3 and PD, and it provides an avenue to comprehend the role of Srpk3 during dopaminergic cell loss and a-syn increase in the brain of PD mice. Furthermore, this study sheds light on the maintenance of Srpk3 as a therapeutic target of PD to control a-syn expression in the pathogenesis of PD.

\section{Declarations}

\section{Funding}

This study was supported by a National Research Foundation of Korea (NRF) grant funded by the Korean Government's Ministry of Science, ICT and Future Planning (MSIP) (No. 2020R1/1A3A04036500).

\section{Acknowledgements}

Not applicable

\section{Competing interest}

The authors declare that they have no competing interests.

\section{Availability of data and material (data transparency)}

Not applicable

\section{Code availability}

Not applicable

\section{Authors' contributions}

SY designed the whole study, interpreted data, performed the protein assay and prepared several samples for immunoblot and immunohistochemistry, and wrote the manuscript. MS performed immunoblot and immunohistochemistry, performed the protein assay and prepared several samples for immunoblot and immunohistochemistry, and interpreted data. All authors have read and approved the manuscript.

\section{Ethics approval}

Not applicable

\section{Consent to participate}

Not applicable 


\section{References}

1. Birkmayer W, Weiler G (1957) On the Pathogenesis of Parkinson's Disease. Nervenarzt 28(2):53-56

2. Tansey MG, Goldberg MS (2010) Neuroinflammation in Parkinson's disease: its role in neuronal death and implications for therapeutic intervention. Neurobiol Dis 37(3):510-518. doi:10.1016/j.nbd.2009.11.004

3. Goedert M, Spillantini MG, Del Tredici K, Braak H (2013) 100 years of Lewy pathology. Nat Rev Neurol 9(1):13-24. doi:10.1038/nrneurol.2012.242

4. Yeo S, An KS, Hong YM, Choi YG, Rosen B, Kim SH, Lim S (2015) Neuroprotective changes in degeneration-related gene expression in the substantia nigra following acupuncture in an MPTP mouse model of Parkinsonism: Microarray analysis. Genet Mol Biol 38(1):115-127. doi:10.1590/S1415-475738120140137

5. Maelicke A, Lubbert $H$ (2002) DEPD, a high resolution gene expression profiling technique capable of identifying new drug targets in the central nervous system. J Recept Signal Transduct Res 22(14):283-295. doi:10.1081/RRS-120014602

6. Xu Y, Yu W, Xiong Y, Xie H, Ren Z, Xu D, Lei M, Zuo B, Feng X (2011) Molecular characterization and expression patterns of serine/arginine-rich specific kinase 3 (SPRK3) in porcine skeletal muscle. Mol Biol Rep 38(5):2903-2909. doi:10.1007/s11033-010-9952-1

7. Choi YG, Yeo S, Hong YM, Kim SH, Lim S (2011) Changes of gene expression profiles in the cervical spinal cord by acupuncture in an MPTP-intoxicated mouse model: microarray analysis. Gene 481(1):7-16. doi:10.1016/j.gene.2011.03.006

8. Lin WJ, Hsueh HM, Chen JJ (2010) Power and sample size estimation in microarray studies. BMC Bioinformatics 11:48. doi:10.1186/1471-2105-11-48

9. Vicente Miranda H, Cassio R, Correia-Guedes L, Gomes MA, Chegao A, Miranda E, Soares T, Coelho M, Rosa MM, Ferreira JJ, Outeiro TF (2017) Posttranslational modifications of blood-derived alphasynuclein as biochemical markers for Parkinson's disease. Sci Rep 7(1):13713. doi:10.1038/s41598017-14175-5

10. Nakagawa O, Arnold M, Nakagawa M, Hamada H, Shelton JM, Kusano H, Harris TM, Childs G, Campbell KP, Richardson JA, Nishino I, Olson EN (2005) Centronuclear myopathy in mice lacking a novel muscle-specific protein kinase transcriptionally regulated by MEF2. Genes Dev 19(17):20662077. doi:10.1101/gad. 1338705

11. Seo MH, Yeo S (2021) Srpk3 Decrease Associated with Alpha-Synuclein Increase in Muscles of MPTP-Induced Parkinson's Disease Mice. Int J Mol Sci 22(17). doi:10.3390/ijms22179375

\section{Figures}




\section{Figure 1}

Dopaminergic cell and alpha-synuclein (a-syn) expression in an MPTP-induced parkinsonism mouse model. The substantia nigra (SN; a, c, d, and f) and striatum (ST; b and e) were immunostained with an anti-tyrosine hydroxylase (TH; a and d, 100x; b and e, 40x) antibody and an anti-a-syn (c and f, 1000x) antibody. Immunoblot analysis (g) showed that the TH expression of SN and ST decreased, whereas the a-syn expression of SN increased in MPTP; these were statistically significant in comparison with the MPTP normalized to $100 \%$ of control (h). CTL, saline control; MPTP, MPTP treated. ${ }^{*} p<0.005$.

\section{Figure 2}

Serine/arginine-rich protein specific kinase 3 (Srpk3) expression decreased in an MPTP-induced 4-week parkinsonism mouse model. (a-f) The results of the immunohistochemistry. (g) The results of the immunoblot assay. (h) Histograms of the microarray and the western-blot analyses represent that Srpk3 expression has decreased in MPTP, compared to the control normalized to $100 \%$. CTL, saline control; MPTP, MPTP treated. ${ }^{*} p<0.005$ and ${ }^{* *} p<0.001$ compared to CTL.

\section{Figure 3}

Serine/arginine-rich protein specific kinase 3 (Srpk3) co-localized with a-synuclein (a-syn) in substantia nigra of an MPTP-induced 4-week parkinsonism mouse model. The cells were immunofluorescently labeled with anti-Srpk3 $(a, e)$ and anti-a-syn ( $b, f)$ antibodies by using rhodamine avidin ( $a, e$; red) and fluorescein avidin (b, f; green). The 'merged' panels (c, g) show merged images of the left $(a, e)$ and middle $(b, f)$ panels, respectively. The 'merged with dapi' panels $(d, h)$ show merged images of the left $(a$, e) and middle (b, $f$ ) panels with dapi, respectively. Note the yellow-colored regions in merged panels indicating the co-localization of the Srpk3 and a-syn. CTL, saline control; MPTP, MPTP treated. Scale bar $=10 \mu \mathrm{m}$.

\section{Figure 4}

Western blot analysis (a) displaying the administration of a serine/arginine-rich protein specific kinase 3 (Srpk3) short interfering RNA (siRNA) leading to a decrease in Srpk3 expression but increase in asynuclein (a-syn) expression in SH-SY5Y cells. (b) Histograms of Srpk3 and a-syn expression were shown in comparison to those of the control group, siRNA 10, and siRNA 100. CTL, control siRNA treatment (100 
nM for 2 days); siSRPK3 10, Srpk3 siRNA treatment (10 nM for 2 days); siSRPK3 100, Srpk3 siRNA treatment (100 nM for 2 days). ${ }^{*} \mathrm{p}<0.05$ and ${ }^{* *} \mathrm{p}<0.005$ compared to CTL.

\section{Figure 5}

Exacerbation induced by a serine/arginine-rich protein specific kinase 3 (Srpk3) short interfering RNA (siRNA) upon 1-methyl-4-phenylpyridinium (MPP+) intoxication in the SH-SY5Y cells. Western blot analysis (a) displaying that in the SH-SY5Y cells, Srpk3 siRNA decreased tyrosine hydroxylase (TH) and increased a-synuclein (a-syn) upon MPP + treatment. (b) Histograms of TH and a-syn expression were shown. CTL, $100 \mathrm{nM}$ negative control siRNA treatment; MPP+, $500 \mu \mathrm{M}$ MPP + treatment; MPP + 10, 10 nM Srpk3 siRNA and $500 \mu \mathrm{M}$ MPP + treatment; MPP + 100, 100 nM Srpk3 siRNA and $500 \mu \mathrm{M}$ MPP + treatment. SH-SY5Y cells were transfected with Srpk3 siRNA. After $6 \mathrm{~h}$, the SH-SY $5 Y$ cells were treated with $500 \mu \mathrm{M}$ MPP + for $18 \mathrm{~h} .{ }^{*} \mathrm{p}<0.05$ was compared to $\mathrm{CTL}$, and $\# \mathrm{p}<0.05$ was compared to MPP +.

\section{Figure 6}

Co-localization of serine/arginine-rich protein specific kinase 3 (Srpk3) with a-synuclein (a-syn) in SHSY5Y cells treated with 1-methyl-4-phenylpyridinium (MPP+). The treated cells were immunostained with anti-Srpk3 (b, f) and anti-a-syn (c, g) antibodies labeled with rhodamine avidin (a, d) and fluorescein avidin $(b, e)$, respectively. The right panels $(d, h)$ show the merged images of the left $(b, f)$ and middle (c, g) panels. The white arrows indicate the co-localization of Srpk3 and a-syn. Control (CTL): no treatment; MPP+: $500 \mu \mathrm{M}$ MPP+ treatment. Scale bar $=10 \mu \mathrm{m}$. 